\title{
Colonoscopically Assisted Laparoscopic Polypectomy-An Alternative to Right Hemicolectomy for Large Right-Sided Benign Polyps*
}

\author{
A. Z. Kaleem ${ }^{\#}$, C. Strachan, L. Whittaker, S. M. Ahmad \\ Department of Colorectal Surgery, Northern Lincolnshire and Goole Hospital NHS Foundation Trust, \\ Scunthorpe General Hospital, Scunthorpe, UK \\ Email: \#ahmed.kaleem@nhs.net
}

Received April 28, 2013; revised May 30, 2013; accepted June 8, 2013

Copyright (C) 2013 A. Z. Kaleem et al. This is an open access article distributed under the Creative Commons Attribution License, which permits unrestricted use, distribution, and reproduction in any medium, provided the original work is properly cited.

\begin{abstract}
Introduction: Laparoscopic assisted colonoscopic polypectomies have been well described in the literature and are well established in surgical practice, for removal of large, inaccessible, or flat based polyps. Laparoscope allows the endoscopist a serosal viewpoint and thus clear indication of perforation, in addition to enhancing endoscopic positioning through colonic mobilisation, facilitating polypectomy. We describe a previously rarely published technique, in which the colonoscope directs the surgeon to polyps and laparoscopy enables wedge resection of benign polyps using Endo GIA staplers. Using this method, the colonoscope provides an intra-luminal view ensuring adequate excision with margins whilst the laparoscope provides intra-peritoneal access for the wedge resection. Methods: This is a case series of 12 patients with large tubulovillous adenomas, found and biopsied at colonoscopy. Under a general anaesthetic, an on table colonoscopy was performed to identify and reassess the polyp, whilst a laparoscopy was performed to excise the polyp via wedge resection, using the endoscopic view as guidance. Results: The polyp was identified and completely resected in our 12 patients. All patients were discharged on the first post-operative day. Of the polyps excised, a focus of adenocarcinoma was detected in one and an adjacent endocrine tumour was found in another patient in histology along with tubulovillous adenoma. Rest were all tubulovillous adenomas only. Conclusion: We propose that this technique should be regarded as an alternative to Right hemicolectomies and difficult endoscopic mucosal resections for large adenomas, and be regarded as a definitive and safe procedure in its own right.
\end{abstract}

Keywords: Colonoscopically Assisted Laparoscopic Polypectomy

\section{Introduction}

Colonoscopic polypectomies are the treatment of choice for most benign colonic polyps, and are the most commonly performed therapeutic intervention in colonoscopy. Polypectomy techniques and established practice has evolved considerably in the last 10 years; providing many options for the varying morphology of colonic polyps.

Snare polypectomies (cold/hot) are performed commonly and routinely for most accessible, pedunculated and small polyps. If a broad based polyp is found, more advanced procedures such as endoscopic mucosal resec-

\footnotetext{
*Source of funding: N/A (Department of Colorectal Surgery, Scunthorpe). Disclosure Statement: Mr Ahmed Zaman Kaleem, Miss Caroline Strachan, Miss Laura Whittaker and Mr Syed Muzaffar Ahmad have no conflicts of interest or financial ties to disclose.

${ }^{\sharp}$ Corresponding author.
}

tions (EMR), endoscopic sub mucosal dissections or formal operative colonic resections are performed to remove them. EMR techniques are growing in popularity given the improved accuracy and safety profiles associated with raising a lesion via injection to facilitate ease of removal and thus identification of an invading lesion. EMR is now recommended by the British Society of Gastroenterology guidelines for flat and depressed lesions. The risk of perforation of the colon during colonoscopic polypectomies varies between $0.1 \%$ and $3 \%$, in some studies [1] and between $0 \%-0.19 \%$ for therapeutic interventions, according to the British society of gastroenterology guidelines [2]. A recent Japanese study has reported figures of $0.91 \%$ perforation risk associated with EMR, compared with $0.17 \%$ with simple Polypectomy and 3.3\% with Endoscopic Mucosal resection [3]. Such figures rise with the size and location of the polyp. 
Perforation risk is greater with polyps $>3 \mathrm{~cm}$ and when located in the thin walled caecum or ascending colon. This is due to the high wall tension in the caecum. In addition there is a risk of thermal transmural damage to the colonic mucosal surface in coagulating the polyp during snare removal.

The well documented and established method of combining laparoscopy with colonoscopy to perform colonoscopic polypectomies, allows the direct visualization of the polyp and the extra-luminal surface with the laparoscope $[4,5]$. Thus if perforation were to occur, the laparoscope allows this to be viewed and immediately repaired, or indeed proceed to laparoscopic resection.

We describe an alternative technique of polypectomy, for benign colonic polyps, utilizing the principles of simultaneous laparoscopy and colonoscopy. We propose the colonoscopically assisted laparoscopic polypectomy as a scarcely described technique and improved method of polypectomy using a laparoscopic wedge resection of the segment of colon containing the benign polyp.

\section{Materials and Methods}

Thus far, we have successfully carried out this procedure on 12 patients, 7 male and 5 female, with an age range between 66 and 92. Pathological examination from initial biopsies showed tubulovillous adenoma in all 12 patients. 7 of the 12 polyps were located in the caecum, and 5 polyps were located in the ascending colon and the patients were referred for this procedure, as the polyps were deemed unsuitable for colonoscopic resection.

The resected polyps ranged in size from $15 \mathrm{~mm}$ in maximum diameter to $50 \mathrm{~mm}$ from colonoscopic assisted laparoscopic polypectomy. The pathology of the wedge resections of the polyps showed tubulovillous adenoma in 11 of the 12 patients and a focus of adenocarcinoma in 1 patient, where the tumour had been completely excised at all margins. This case was taken to the multi-disciplenary team meeting (MDT) where the decision to perform a laparoscopic Right hemicolectomy was taken. In one patient, the appendix was removed in the wedge resection given the proximity of the appendiceal opening to the polyp. The pathological analysis of the polyp was a completely excised TVA; however, the pathological analysis of the appendix was that of a well differentiated endocrine tumour, confined to the sub mucosa, and a pathological staging of pT1, completely excised. Thus, histological analysis confirms complete excision of adenoma in all performed polypectomies.

\section{Technique}

The patient receives full bowel preparation for colonoscopy. The patients are admitted the day before surgery and are consented and counselled for colonoscopically assisted laparoscopic polypectomy, +/- $\mathrm{R}$ hemicolectomy (laparoscopic/ open), with all the potential risks involved in such a procedure for example; leak, need for further surgery if malignancy is suspected or confirmed on histology, incomplete resection, further polyps, anaesthetic risks. It is explained to the patient that this is a novel procedure in this country, and permission is taken for publication and photography during laparoscopy/ colonoscopy.

The patient is positioned in the lithotomy position on the operating table, after a general anaesthetic is administered, prepped and draped for a laparoscopic procedure. A colonoscopy is performed and the polyp is identified with the colonoscope. A tattoo may also be used to facilitate polyp identification at laparoscopy. At this point, laparoscopy is performed, via a $10 \mathrm{~mm}$ umbilical port. A further 2 ports are inserted under vision, one $12 \mathrm{~mm}$ port in the hypo gastric and another $5 \mathrm{~mm}$ port in the paraumblical region as shown in Figure 1.

Tattoo on bowel or light of colonoscope is used to find polyp from abdominal side (Figures 2(a) and 3). If visualization from abdominal side via laparoscope is difficult or polyp is located posteriorly then caecum and ascending colon are mobilized from lateral aspect. When polyp is identified both endoscopically and laparoscopically then by using an endoscopic GIA linear stapler, the segment of bowel containing the polyp is stapled off (Figures 2(b), 4 and 5). Before firing the stapler using colonoscope, it is made sure that polyp is completely excised. Once divided it is removed in a Burt bag through the umbilical port (Figure 6). The role of the colonoscopy here is to directly visualize the lumen to identify that the polyp is being completely enveloped by the stapler along with good margins. The stapler will not be closed and fired before the surgeon is satisfied with the colonoscopic image demonstrating the complete

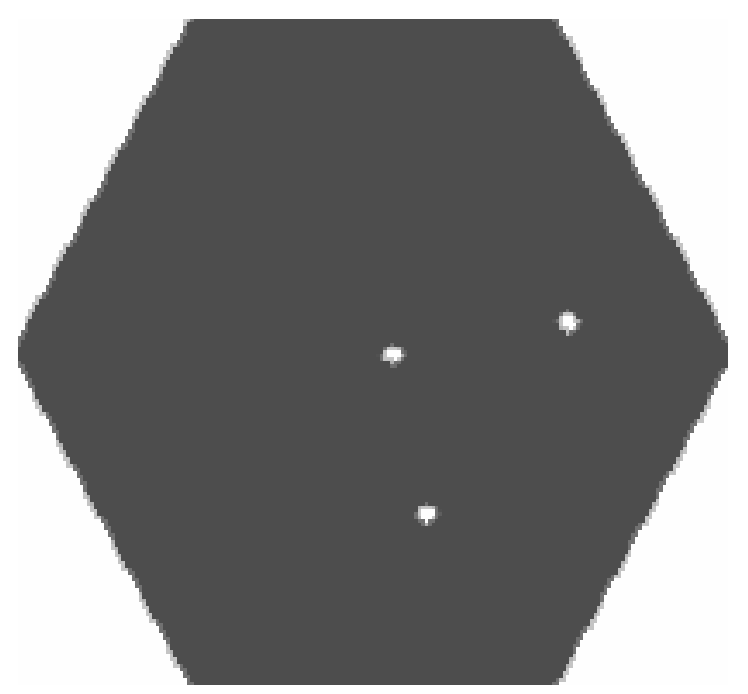

Figure 1. Port positioning. 


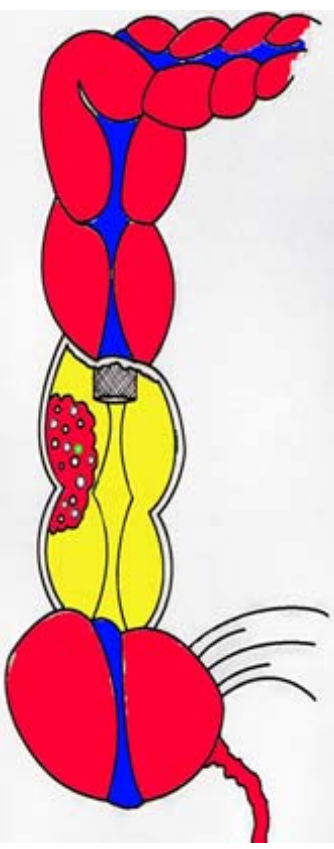

(a)

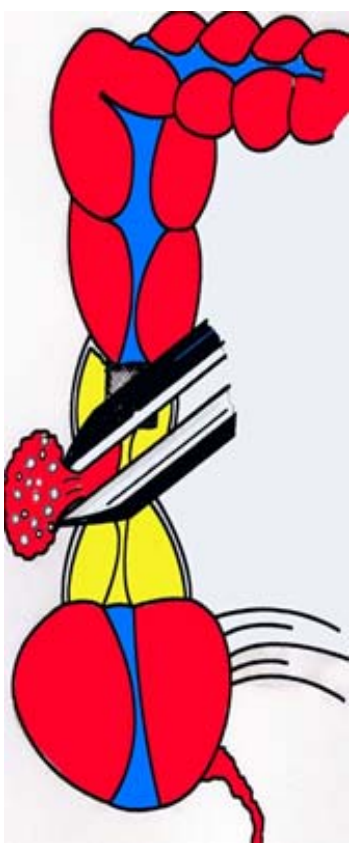

(b)
Figure 2. (a) Demonstration of the right sided polyp; (b) Demonstration of the wedge resection.

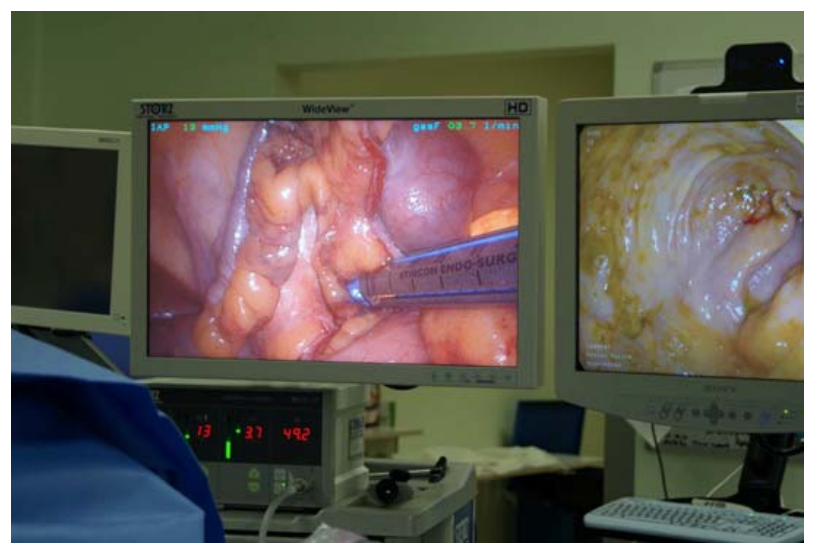

Figure 3. Double view of linear stapler and colonoscopic view.

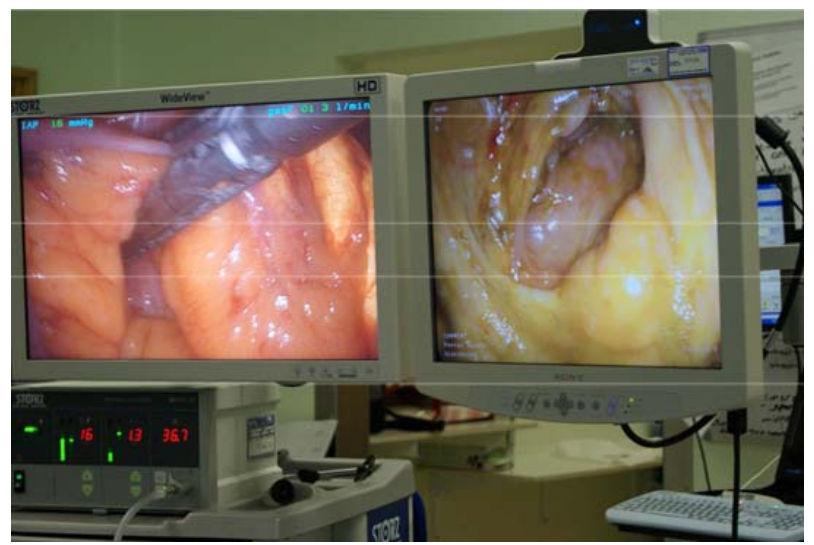

Figure 4. Firing of the stapler and the colonoscopic view.

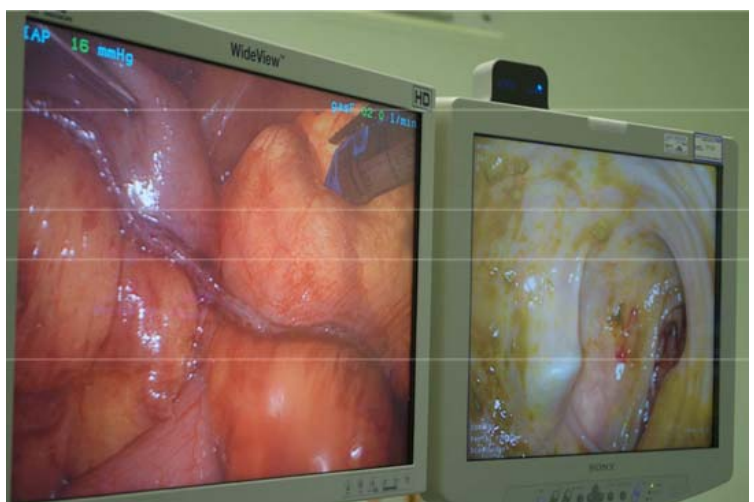

Figure 5. Post wedge stapling of the polyp.

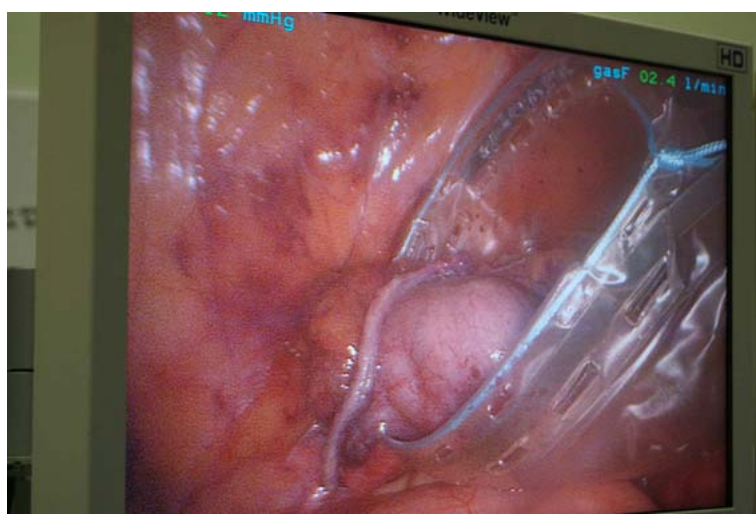

Figure 6. Retrieval of the wedge of caecum in a Burt bag.

involvement of the polyp in the stapler. The right colon has, in 11 out of our 12 cases not required mobilization in order to carry out the segmental resection. In one case, the polyp was located on the posterior wall and thus the right colon had to be laparoscopically mobilized in order to angle the laparoscopic stapler to achieve polypectomy.

The colonoscope is then withdrawn with intermittent suction to deflate the colon, and the ports are removed under direct vision and the wounds are closed in the usual manner. Patients remain in hospital overnight for monitoring and are discharged the following day if well. All patients were discharged the following day.

Colonoscopically assisted laparoscopic polypectomy was successfully achieved in 12 cases. All patients were able to tolerate oral intake following the procedure and there were no complications. The pathological analysis has been detailed above. Of the excised polyps, 11 were confirmed as benign adenomatous polyps and 1 polyp showed a focus of moderately differentiated adenocarcinoma arising from Tubulovillous adenomatous lesion. This patient went on to have a laparoscopically assisted $\mathrm{R}$ hemicolectomy.

\section{Discussion}

Adenomatous colonic polyps are amongst the most 
common disorders of the colon and regarded as pre-malignant conditions. The majority of colonic polyps are amenable to endoscopic snare excision. Large flat or inaccessible right sided polyps pose a problem to safe endoscopic excision. The danger of incomplete excision or perforation of the colon is significant, particularly in thin walled caecum and ascending colon, and varies amongst reports to as much as 3\% [2]. Laparoscopic assisted colonoscopic polypectomies are now well established for the removal of such polyps and provide the extra security of an intra-abdominal, serosal view of the colon whilst the endoscopist is removing the polyp via snare [5]. Thus in the case of perforation, it may be identified and treated immediately. In addition, the use of the colonoscope allows for accurate location of the polyp and thus minimizing the need for colonic mobilization, with the exception of posterior/lateral wall located polyp. In this approach, the polyp is removed piecemeal and without surrounding margins and thus histological comments on the complete excision are difficult. There is also the consideration of a late perforation due to diathermy injury which may not be evident at the time of laparoscopy/ colonoscopy. We describe an alternative technique to ensure complete polyp and margin removal, as a simple wedge resection with the combination of laparoscopy and colonoscopy. This technique has previously been described, in association with the laparoscopic assisted EMR, but not as a definitive procedure in its own right $[6,7]$. Using an endoscopic GIA stapler, guided by the colonoscope, a wedge of the affected part of colon is removed with adequate margins. Laparoscopic tools are used to lift up the colon to achieve appropriate stapler angle, and the colonoscope provides simultaneous view of the polyp to the laparoscopic surgeon, thus ensuring complete removal of the polyp. This procedure for benign colonic polyps eliminates the risk of perforation in difficult polypectomies whilst also providing complete excision with margins. This procedure has been described previously in the literature as an alternative to laparoscopically assisted colonoscopic excision, depending upon the location of the polyp. Due to the fragility of this caecal walls, we believe that the endoscopic wedge resection of tubulovillous adenomas in the caecum or ascending colon should be considered as a safer and more definitive procedure within its own right.

The negative caveat to the combined laparoscopic and colonoscopic polyp removals is the potential need for definitive resection if histology shows malignant change. 1 of the 12 patients in our experience thus far has had a focus of adenocarcinoma in the resected polyp and has thus required formal laparoscopic hemicolectomy. Thus, the patient undergoes 2 general anaesthetics and laparoscopic abdominal surgeries within a short time frame.

However, given the common place nature of colonic polyps, and the advent of bowel screening, a large number of benign polyps are being identified that are not amenable to endoscopic resection. We feel that in such circumstances colonoscopic assisted laparoscopic polypectomies provide an excellent, safe and definitive excision method, particularly for right sided lesions. Patients may go home the following day, and may eat and drink once recovered from the anaesthetic.

\section{Conclusion}

Colonoscopically assisted laparoscopic polypectomies provide an alternative and safe method of colonic polypectomy for benign polyps, which are not amenable to endoscopic resection. Laparoscopic wedge resection ensures complete polypectomy with margins, and avoids formal laparoscopic colonic resection and the risk of perforation in difficult inaccessible polyps with endoscopic mucosal resection. We recommend this should be considered as a definitive procedure for benign polyps, with no histological evidence of malignant transformation, and propose this technique as an alternative to right hemicolectomies for benign lesions.

\section{REFERENCES}

[1] M. E. Franklin, A. Leyva-Alvizo, et al., "Laparoscopically Monitored Colonoscopic Polypectomy: An Established Form of Endoluminal Therapy for Colorectal Polyps," Surgical Endoscopy, Vol. 21, No. 9, 2007, pp. 1650-1653. doi:10.1007/s00464-007-9237-5

[2] British Society of Gastroenterology Guidelines, "Complications of Colonoscopy," 2006. www.bsg.org

[3] S. Oka, et al., "Current Status in the Occurrence of Post Operative Bleeding, Perforation, and Local/Residual Recurrence during Colonoscopic Treatment in Japan," Digestive Endoscopy, Vol. 22, No. 4, 2010, pp. 376-380. doi:10.1111/j.1443-1661.2010.01016.x

[4] C. Hensman, A. J. Luck and P. J. Hewett, "Laparoscopic Assisted Colonoscopic Polypectomy," Surgical Endoscopy, Vol. 13, No. 3, 1999, pp. 231-232. doi:10.1007/s004649900951

[5] M. E. Franklin, J. A. Diaz-E, et al., "Laparoscopic-Assisted Colonoscopic Polypectomy: The Texas Endosurgery Institute Experience," Diseases of the Colon and Rectum, Vol. 43, No. 9, 2000, pp. 1246-1249. doi:10.1007/BF02237429

[6] C. J. A. Bowles, R. Leicester et al. "A Prospective Study of Colonoscopy Practice in the UK Today, Are We Prepared for National Colorectal Cancer Screening Tomorrow," Gut, Vol. 53, No. 2, 2004, pp. 277-283. doi:10.1136/gut.2003.016436

[7] D. Wilhelm, S. Von Delius, et al., "Combined Laparoscopic- endoscopic Resections of Colorectal Polyps: 10 Year Experience and Follow Up," Surgical Endoscopy, Vol. 23, No. 4, 2009, pp. 688-693. doi:10.1007/s00464-008-0282-5 